\title{
Self-balance Motion and Appearance Model for Multi-object Tracking in UAV
}

\author{
Hongyang $\mathrm{Yu}$ \\ Harbin Institute of Technology \\ hyang.yu@hit.edu.cn
}

\author{
Guorong Li \\ University of Chinese Academy of \\ Sciences \\ liguorong@ucas.ac.cn
}

\author{
Weigang Zhang \\ Harbin Institute of Technology, \\ Weihai \\ wgzhang@hit.edu.cn
}

\author{
Hongxun Yao \\ Harbin Institute of Technology \\ h.yao@hit.edu.cn
}

\begin{abstract}
Under the tracking-by-detection framework, multi-object tracking methods try to connect object detections with target trajectories by reasonable policy. Most methods represent objects by the appearance and motion. The inference of the association is mostly judged by a fusion of appearance similarity and motion consistency. However, the fusion ratio between appearance and motion are often determined by subjective setting. In this paper, we propose a novel self-balance method fusing appearance similarity and motion consistency. Extensive experimental results on public benchmarks demonstrate the effectiveness of the proposed method with comparisons to several state-of-the-art trackers.
\end{abstract}

\section{KEYWORDS}

Multi-object tracking, Neural networks, UAV

\section{ACM Reference Format:}

Hongyang Yu, Guorong Li, Weigang Zhang, Hongxun Yao, and Qingming Huang. 2019. Self-balance Motion and Appearance Model for Multi-object Tracking in UAV. In ACM Multimedia Asia (MMAsia '19), December 15-18, 2019, Beijing, China. ACM, New York, NY, USA, 6 pages. https://doi.org/10.1145/3338533.3366561

\section{INTRODUCTION}

Multi-object tracking in videos is an important task within the field of computer vision. Multi-object tracking aims to find all the object trajectories in videos or image sequences. Thanks to the advancement of the object detection methods in recent years, such as DPM[9], SDP[33], Faster r-cnn[25] and YOLO $[23]$, the tracking-by-detection framework [5, 10,

Permission to make digital or hard copies of all or part of this work for personal or classroom use is granted without fee provided that copies are not made or distributed for profit or commercial advantage and that copies bear this notice and the full citation on the first page. Copyrights for components of this work owned by others than ACM must be honored. Abstracting with credit is permitted. To copy otherwise, or republish, to post on servers or to redistribute to lists, requires prior specific permission and/or a fee. Request permissions from permissions@acm.org.

MMAsia '19, December 15-18, 2019, Beijing, China

(C) 2019 Association for Computing Machinery.

ACM ISBN 978-1-4503-6841-4/19/12 . .\$15.00

https://doi.org/10.1145/3338533.3366561
Qingming Huang

Harbin Institute of Technology,

University of Chinese Academy of

Sciences

qmhuang@ucas.ac.cn
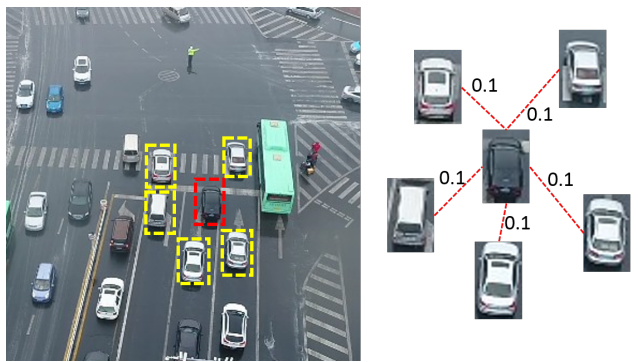

Effective

Appearancediscriminating

Object
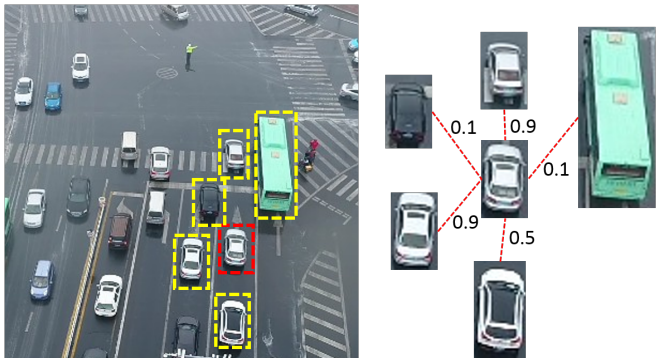

Confusing

Appearance-

discriminating

Object

Figure 1: Schematic of the effectiveness of appearancediscriminating. The top target can be discriminated with the objects around by appearance. The bottom target is confusing with the objects around only by appearance. The edge between the objects shows the appearance similarity between them.

$17,29,31]$ is widely used in multi-object tracking task. This framework has advantages that the number of objects in scene is self-adaptive and object detection results offer much more accurate object positions for multi-object tracking methods.

According to the way of using object detections, the tracking-by-detection based multi-object tracking methods could be broadly categorized into two groups: the global(batch) methods such as $[5,29,31]$ and the online methods such as $[10,17]$. The global methods utilize the whole object detections in sequences, finding the global optimal association between all detections. So in each frame, these methods associate the detections taking into account not only past frames but also the frames in future. In contrast, the online methods 
utilize the object detections in current frame and the trajectories in past frames. Because of lack of information in future frames, the online tracking methods are more challenging with appearance changes of objects and complexity of the context in scene. On the other hand, the online process of these methods corresponds with the reality applications, such as the unmanned aerial vehicle and intelligent surveillance. In this paper, we focus on the online multi-object tracking methods.

Once the detection are known, the trackers would utilize several cues to generate the affinity between the detections and targets. The affinity is general generated by some cues, such as appearance and motion. However, the weight ratio of appearance or motion in the affinity is usually designed by handcrafted. The empirical weight is unified set in the methods. But for each object in the scene,the discriminative ability of appearance or motion varies is various. In Fig. 1, we show the appearance similarity between one target and the objects around it. It is observed that the target on the top could be easily distinguish only by appearance while the targets on the bottom have very similar appearance. Thus, for the target on the bottom, the motion cue should play a more important role for recognizing the target. Meanwhile, the changes of objects and scene background are smooth in the adjacent frames. It means that the ratio of effectiveness between the appearance similarity and motion consistency should be stable. Inspired by this observation, we proposed a novel self-balance method for balancing appearance similarity and motion consistency in distinguishing targets. We utilize the appearance similarity between the targets in the scene for self-adaption of allocating the weights.

In evaluations, we apply the proposed method over a set of challenging sequences that recorded by the Unmanned Aerial Vehicles (UAVs). Results showed that our method can achieve better tracking performance.

\section{RELATED WORKS}

Under the tracking-by-detection framework, most tracking methods [12] contain two main steps: object description and data association. For object description, different features in various cues [35], (such as appearance, motion, shape and context) are utilized to describe each object. For data association, probabilities of connecting detections are computed with the features of the detections extracted in object description step $[19,30]$.

For a better affinity between objects, most methods focus on how to describe the objects. For the appearance description, small pattern is utilized for describing the object patch in some methods. The objects are described by segmenting objects into parts $[11,28]$. In [16], the author utilizes super-pixels to compensate the appearance diversity by the imprecise detections. [36] utilizes Dual Matching Attention Networks to focus on the effective parts of the objects for distinguishing. Some methods utilize person re-identification method enhancing the appearance representation, such as in [31] or use a large convolutional neural networks to associate object detections such as Quad-CNN in [29]. For the motion description, LSTM networks are used for predicting the position of the targets in [18]. [24] exploits two adjacent frames to learn the movement of the target.

The appearance and the motion description are used separately or fused by weights in most methods. There are also several methods merging appearance and motion by connecting the features in the network, such as [13]. However, these methods fuse the cues in the fixed way ignoring the influence of changing context for the discrimination of descriptions. In this paper, we consider about the changing discrimination and propose a novel self-balance method fusing appearance similarity and motion consistency.

\section{SELF-BALANCE MODEL FOR MULTI-OBJECT TRACKING}

The online multi-object tracking methods aim to connect the detections in current frame with the trajectories of the targets. Thus, the detections and past trajectories of targets are once known, the tracking problem can be formulated as

$$
\begin{gathered}
F=\arg \max _{A_{t-1, t}} P\left(\mathbf{D}^{t} \mid \mathbb{T}^{t-1}\right) \\
=\arg \max _{A_{t-1, t}} \sum_{i \in M} \sum_{j \in N \cup\{0\}} a^{i, j} \Omega_{i, j}^{t}, \\
\text { s.t. } \sum_{i \in M, j \neq 0} a^{i, j} \leq 1 \bigwedge \sum_{j \in N \cup\{0\}} a^{i, j}=1, \text { for all } i
\end{gathered}
$$

where $P(\mathbf{D} \mid \mathbb{T})$ is the score of association scheme $A_{t-1, t}$. $a^{i, j}$ is an element of the association matrix $A$, with a binary value $\{1,0\} . i \in M$ represents target $i$ in the target set $\mathbb{T}^{t-1}$, and $j \in N$ corresponds to detection $j$ in the set $\mathbf{D}^{t}$ of detections in the current frame. $\Omega_{i, j}^{t}$ is an affinity function that represents the probability that target $i$ should be combined with detection $j$.

In the original method, $\Omega_{i, j}^{t}$ integrates appearance similarity, motion consistency and shape consistency, i.e.

$$
\Omega_{i, j}^{t}=\sum w_{c} * f_{c}(i, j), c \in\{A P P, M O V, S H A P E \ldots\} .
$$

If some cues are unused in the method, the corresponding $f_{c}(i, j)=0 . w_{c}$ is the weight of the corresponding cue.

In our method, we utilize the appearance similarity and motion consistency to generate the affinity between objects. $\Omega_{i, j}^{t}$ is defined as

$$
\Omega_{i, j}^{t}=w_{i}^{t-1} * f_{M O V}(i, j)+\left(1-w_{i}^{t-1}\right) * f_{A P P}(i, j),
$$

where $f_{A P P}(i, j)$ is appearance similarity between object detections and targets and $f_{M O V}(i, j)$ is the motion consistency. $w^{t-1}$ is the weight learned from the last frame.

The appearance similarity $f_{A P P}(i, j)$ is defined as

$$
f_{A P P}(i, j)=\frac{\mathbf{x}_{i} \cdot \mathbf{x}_{j}}{\left\|\mathbf{x}_{i}\right\| \cdot\left\|\mathbf{x}_{j}\right\|},
$$

$f_{A P P}(i, j)$ is the cosine distance between the feature $\mathbf{x}_{i}$ of target $i$ frame $t-1$ and the feature $\mathbf{x}_{j}$ of the object detection $j$ in frame $t$. 


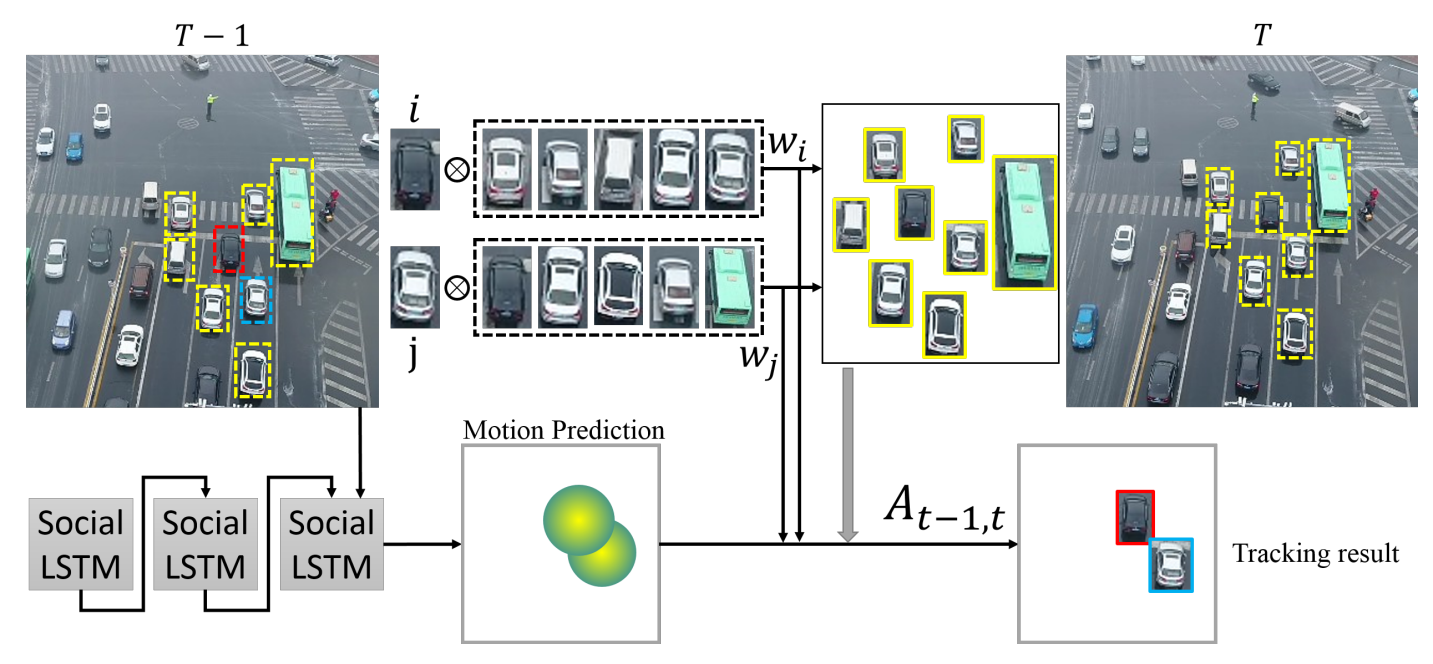

Figure 2: The framework of the proposed method for online multiple object tracking with our self-balance motion and appearance model. In the last frame, the appearance similarity between the target and other targets around it is calculated for generating the weights for each target. Using the Social LSTM network, we could get the motion prediction of targets. Then the affinity $A_{t-1, t}$ between targets and objects in current frame is generated using the weighted appearance similarity and motion prediction.

The motion consistency $f_{M O V}(i, j)$ is defined as

$$
f_{M O V}(i, j)=p\left((x, y)_{j} \mid N_{i}(t-1)\right),
$$

where $N_{i}(t-1)$ is the distribution of the position prediction of target $i$ in frame $t$ from last $t-1$ frames. $p\left((x, y)_{j} \mid N_{i}(t-1)\right)$ is the probability that target $i$ is at the position $(x, y)_{j}$.

As the context information could influence the discrimination of appearance and motion, we generate the weight $w_{i}^{t-1}$ from the context of target $i$ in the last frame. The targets in the area around the target $i$ are regarded as the context. Thus $w_{i}^{t-1}$ is defined as

$$
w_{i}^{t-1}=\sin \left(\frac{\pi}{4} *\left(\max \left(f_{A P P}(i, r)\right)\right)\right), r \in R_{i}^{t-1},
$$

where $f_{A P P}\left(\mathbf{x}_{i}, \mathbf{x}_{r}\right)$ is the cosine distance between the feature $\mathbf{x}_{i}$ of target $i$ in frame $t-1$ and the feature $\mathbf{x}_{r}$ of the targets $R_{i}^{t-1}$ in the area in frame $t-1 . R_{i}^{t-1}$ is the set of objects around the target $i$ at $t-1$ time. If there is some similar target around the target $i$, the weight of motion would be large while the weight of appearance changing small.

The framework schematic of our method is shown in Fig. 2. For each target in frame $t-1$, the weights of the targets are generated by calculating the appearance similarity between the target and others targets around. The appearance feature of target $\mathbf{x}$ is generated by a object re-identification networks [32]. With the weights, the appearance similarity between targets in frame $t-1$ and the objects in frame $t$ would be generated. Then a social LSTM network [1] is introduced for motion prediction. Once the motion prediction and appearance similarity are known, the affinity $\Omega_{i, j}^{t}$ could be calculated using Function. 3 .

Considering about target exit or occlusion, we include a sink, i.e., target 0 , to generate an exit association for each real target. Then $\Omega_{i, 0}$ is set to a value in $(0,1)(0.01$ in this study) for the missing target. The optimal association scheme $A_{t-1, t}$ is worked out using an association method, e.g., the Hungarian algorithm ([21]). Finally the tracking results in current frame are achieved.

\section{EXPERIMENTS}

We evaluate our self-balance motion and appearance method (denoted by SBMA) together with 8 recent algorithms including online methods ( RMOT [34], RLSTM [18], SORT [3] and SLSTM [1]) and batch-based methods (CEM [20], GOG [22], IOUT [4] and SMOT [7]).

Metrics. To evaluate the performance of MOT methods, we use multiple metrics, including Identification Precision (IDP) [27], Identification Recall (IDR), and the corresponding F1 score called IDF1 (the ratio of correctly identified detections to the average number of ground-truth and computed detections). Multiple Object Tracking Accuracy (MOTA) [2], Multiple Object Tracking Precision (MOTP), Mostly Tracked targets (MT, defined as more than $80 \%$ of trajectories being covered by the ground-truth), Mostly Lost targets (ML, defined as less than $20 \%$ of trajectories being covered by the ground-truth), the number of False Positives (FP), the number of False Negatives (FN), the number of ID Switches (IDS) and the number of times a trajectory is Fragmented (FM) are also considered.

Dataset. We evaluate our method on the dataset UAVDT [8]. The dataset offers 50 sequences recorded from Unmanned Aerial Vehicles (60\% for training and $40 \%$ for testing). We compare our method with 8 recent algorithms on the testing sequences.

Implementation. We implemented our framework in Tensorflow1.0 on a computer with an i7 $6700 \mathrm{CPU}$, an NVIDIA Titan X GPU and 32 GB of RAM. For the re-identification 
Detection Input: Faster-RCNN [26]

\begin{tabular}{|c|c|c|c|c|c|c|c|c|c|c|c|}
\hline MOT methods & IDF1 & IDP & IDR & MOTA & MOTP & $\mathrm{MT}[\%]$ & ML[\%] & FP & $\mathrm{FN}$ & IDS & FM \\
\hline CEM [20] & 10.2 & 19.4 & 7.0 & -7.3 & 69.6 & 7.3 & 68.6 & 72,378 & 290,962 & 2,488 & 4,248 \\
\hline GOG [22] & 18.0 & 23.3 & 14.6 & 34.4 & 72.2 & 35.5 & 25.3 & 41,126 & 168,194 & 14,301 & 12,516 \\
\hline IOUT [4] & 23.7 & 30.3 & 19.5 & 36.6 & 72.1 & 37.4 & 25.0 & 42,245 & 163,881 & 9,938 & 10,463 \\
\hline RMOT [34] & 33.3 & 27.8 & 41.4 & -39.8 & 72.3 & 36.7 & 25.7 & 319,008 & 151,485 & 5,973 & 5,897 \\
\hline RLSTM [18] & 31.3 & 38.6 & 26.3 & 25.6 & 69.1 & 36.7 & 25.7 & 71,955 & 180,461 & 1,333 & 13,088 \\
\hline SMOT [7] & 45.0 & 55.7 & 37.8 & 33.9 & 72.2 & 36.7 & 25.7 & 57,112 & 166,528 & 1,752 & 9,577 \\
\hline SORT [3] & 43.7 & 58.9 & 34.8 & 39.0 & 74.3 & 33.9 & 28.0 & 33,037 & 172,628 & 2,350 & 5,787 \\
\hline SLSTM [1] & 37.2 & 46.8 & 30.8 & 37.9 & 72.0 & 38.2 & 24.4 & 44,783 & 161,009 & 6,048 & 12,051 \\
\hline SBMA & 48.5 & 61.1 & 40.3 & 38.6 & 72.1 & 38.9 & 24.4 & 44,724 & 160,950 & 3,489 & 11,796 \\
\hline \multicolumn{12}{|c|}{ "Detection Input: R-FCN [6] } \\
\hline MOT methods & IDF1 & IDP & IDR & MOTA & MOTP & $\mathrm{MT}[\%]$ & ML[\%] & FP & $\mathrm{FN}$ & IDS & FM \\
\hline CEM [20] & 10.3 & 18.4 & 7.2 & -9.6 & 70.4 & 6.0 & 67.8 & 81,617 & 289,683 & 2,201 & 3,789 \\
\hline GOG $[22]$ & 30.9 & 38.8 & 25.6 & 28.5 & 77.1 & 34.4 & 28.6 & 60,511 & 176,256 & 6,935 & 6,823 \\
\hline IOUT [4] & 44.0 & 47.5 & 40.9 & 26.9 & 75.9 & 44.3 & 22.9 & 98,789 & 145,617 & 4,903 & 6,129 \\
\hline RMOT [34] & 34.4 & 28.7 & 43.0 & -43.8 & 76.3 & 36.7 & 25.7 & 328,677 & 158,760 & 2,949 & 2,286 \\
\hline RLSTM [18] & 29.3 & 34.8 & 25.2 & 14.7 & 70.6 & 36.7 & 25.7 & 97,670 & 191,720 & 1,395 & 9,953 \\
\hline SMOT [7] & 44.0 & 53.5 & 37.3 & 24.5 & 77.2 & 33.7 & 29.2 & 76,544 & 179,609 & 1,370 & 5,142 \\
\hline SORT [3] & 42.6 & 58.7 & 33.5 & 30.2 & 78.5 & 29.5 & 31.9 & 44,612 & 190,999 & 2,248 & 4,378 \\
\hline SLSTM [1] & 35.6 & 43.6 & 30.1 & 27.5 & 76.9 & 35.2 & 27.7 & 66,980 & 172,942 & 7,355 & 9,791 \\
\hline SBMA & 46.9 & 57.5 & 39.6 & 28.5 & 76.9 & 35.0 & 27.6 & 67,134 & 173,096 & 3,367 & 9,759 \\
\hline \multicolumn{12}{|c|}{ "Detection Input: SSD [15] } \\
\hline MOT methods & IDF1 & IDP & IDR & MOTA & MOTP & $\mathrm{MT}[\%]$ & $\mathrm{ML}[\%]$ & $\mathrm{FP}$ & $\mathrm{FN}$ & IDS & FM \\
\hline CEM [20] & 10.1 & 21.1 & 6.6 & -6.8 & 70.4 & 6.6 & 74.4 & 64,373 & 298,090 & 1,530 & 2,835 \\
\hline GOG $[22]$ & 29.2 & 33.6 & 25.9 & 33.6 & 76.4 & 36.0 & 22.4 & 70,080 & 148,369 & 7,964 & 10,023 \\
\hline $\operatorname{IOUT}[4]$ & 29.4 & 34.5 & 25.6 & 33.5 & 76.6 & 34.3 & 23.4 & 65,549 & 154,042 & 6,993 & 8,793 \\
\hline RMOT [34] & 26.1 & 18.9 & 41.8 & -101.0 & 75.1 & 36.7 & 25.7 & 544,591 & 131,382 & 9,252 & 7,211 \\
\hline RLSTM [18] & 26.5 & 28.9 & 24.4 & 7.6 & 69.0 & 36.7 & 25.7 & 129,660 & 182,828 & 2,347 & 12,654 \\
\hline SMOT $[7]$ & 41.9 & 45.9 & 38.6 & 27.2 & 76.5 & 34.9 & 22.9 & 95,737 & 149,777 & 2,738 & 9,605 \\
\hline SORT [3] & 37.1 & 45.8 & 31.1 & 33.2 & 76.7 & 27.3 & 25.4 & 57,440 & 166,493 & 3,918 & 7,898 \\
\hline SLSTM [1] & 39.4 & 44.2 & 35.5 & 33.0 & 75.8 & 37.3 & 21.6 & 77,706 & 144,617 & 6,019 & 13,332 \\
\hline SBMA & 43.1 & 48.3 & 38.8 & 33.6 & 75.8 & 37.7 & 21.4 & 77,299 & 144,191 & 4,932 & 13,468 \\
\hline \multicolumn{12}{|c|}{ " Detection Input: RON [14] } \\
\hline MOT methods & IDF1 & IDP & IDR & MOTA & MOTP & $\operatorname{MT}[\%]$ & ML[\%] & FP & FN & IDS & FM \\
\hline CEM [20] & 10.1 & 18.8 & 6.9 & -9.7 & 68.8 & 6.9 & 72.6 & 78,265 & 293,576 & 2,086 & 3,526 \\
\hline GOG [22] & 51.0 & 60.2 & 44.3 & 35.7 & 72.0 & 43.9 & 26.2 & 62,929 & 153,336 & 3,104 & 5,130 \\
\hline $\operatorname{IOUT}[4]$ & 50.1 & 59.1 & 43.4 & 35.6 & 72.0 & 43.9 & 26.2 & 63,086 & 153,348 & 2,991 & 5,103 \\
\hline RMOT [34] & 28.8 & 22.5 & 40.0 & -70.0 & 70.9 & 36.7 & 25.7 & 418,222 & 153,480 & 7,902 & 7,007 \\
\hline RLSTM [18] & 36.5 & 42.4 & 32.1 & 24.1 & 68.7 & 36.7 & 25.7 & 87,589 & 169,866 & 1,156 & 12,657 \\
\hline SMOT [7] & 52.6 & 60.8 & 46.3 & 32.8 & 72.0 & 43.4 & 27.1 & 73,226 & 154,696 & 1,157 & 4,643 \\
\hline SORT [3] & 54.6 & 66.9 & 46.1 & 37.2 & 72.2 & 40.8 & 28.0 & 53,435 & 159,347 & 1,369 & 3,661 \\
\hline SLSTM [1] & 48.1 & 56.4 & 41.9 & 35.0 & 71.9 & 44.5 & 26.2 & 65,093 & 152,481 & 4,013 & 6,059 \\
\hline SBMA & 52.4 & 61.4 & 45.7 & 34.8 & 71.9 & 44.0 & 26.4 & 65,760 & 153,148 & 3,195 & 6,003 \\
\hline
\end{tabular}

Table 1: Results of a quantitative comparison among MOT methods in the testing set of the UAVDT dataset. The best performer and our methods are highlighted in bold.

networks [32] and social LSTM network [1], we use the implementations released by the authors.

Evaluation. For overall evaluation, we utilize object detection results as the inputs for tracking-by-detection methods by four kinds of methods (i.e., Faster-RCNN [26], R-FCN [6], SSD [15] and RON[14]). The tracking results with the four kinds of detections are shown in Table. 1. From the table, it is observed that our method achieves the best results with three of four kinds of detections on the primary metric IDF1 while the tracking results with detections by RON are comparable with the best result. For our method, the performances with different detections are fluctuating smoothly. With the detections by RON, the results of most methods are much better than with other detections. And the performance difference of various methods are smaller with the detection input by RON than by the other detection methods. Compared with 


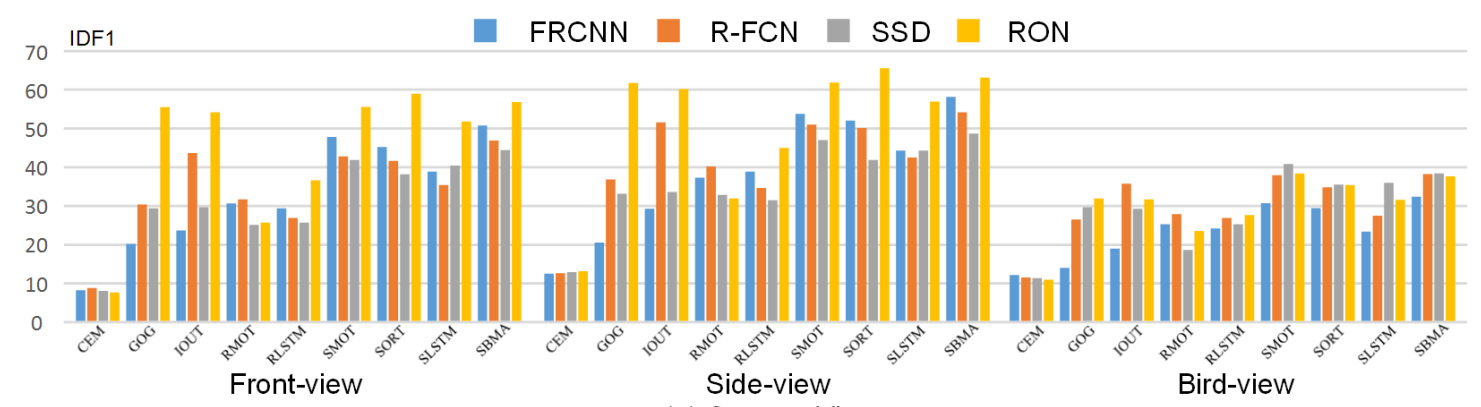

(a) Camera View

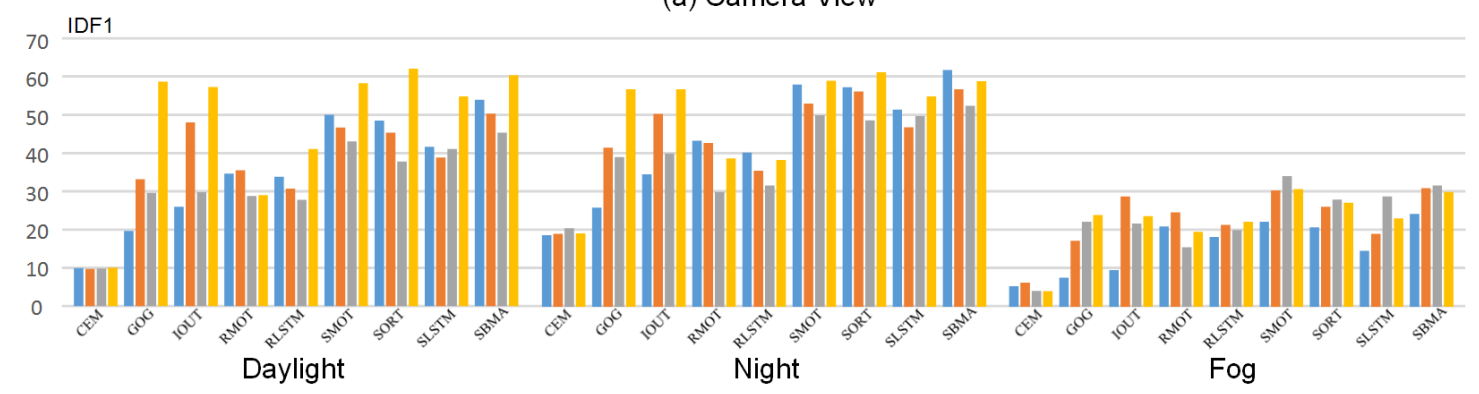

(b) Weather Condition

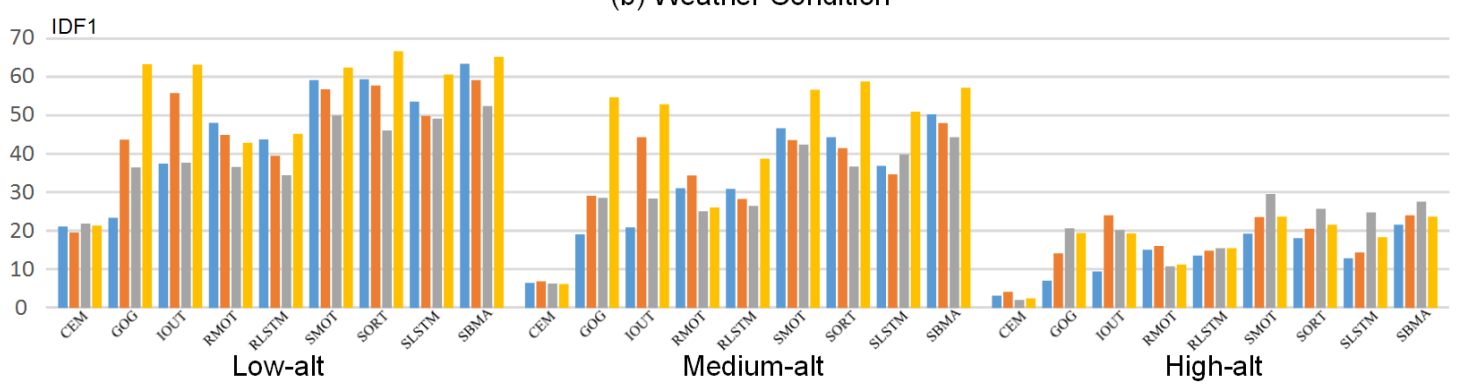

(c) Flying Altitude

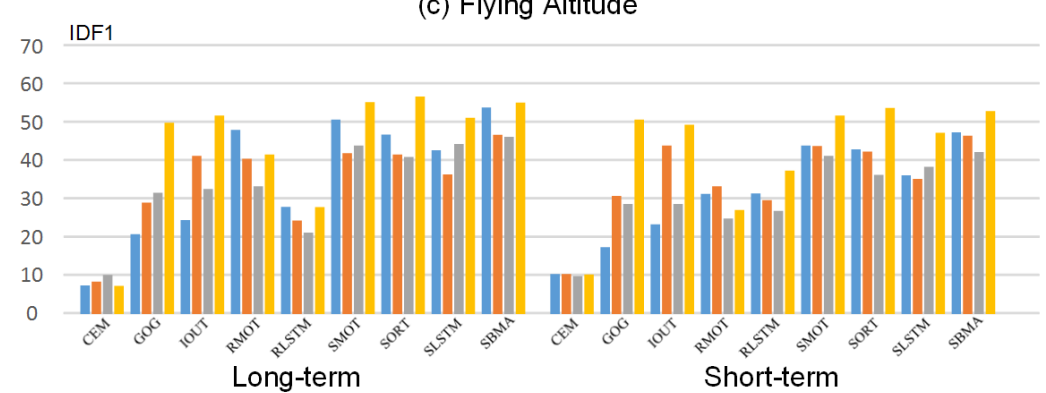

(d) Duration

Figure 3: Results of a quantitative comparison among MOT methods for each attribute.

the original SLSTM, our method has more improvement on most metrics. Especially, with less IDS and FM, our method could generate much more integrated trajectories. It means that using our self-balance method, the affinity between objects are more robust. The higher IDF1 score also reflects the effectiveness of our method.

We also evaluate the performance of our methods on various attributes. The UAVDT dataset offer four kinds of attributes, i.e., Camera View (view of the camera on UAV),
Weather Condition (illumination when videos are captured), Flying Altitude (maximum flying altitude of UAV when the videos are captured) and Duration (the length of a sequence). The results of the quantitative comparison among MOT methods for each attribute are shown in Fig. 3. For Camera View, most methods perform better in front-view and side-view than in the bird-view, as shown in Fig. 3(a), because these views offer more details of the objects for detection and tracking. Our method achieves better results with 
the larger appearance difference between objects in frontview and side-view. For Weather Condition, as shown in Fig. 3(b), most methods perform better in day and night, especially with the RON detection as detection input. Our proposed methods mostly perform best in these condition. Due to poor illumination, the performance of most methods declines in fog. For Flying Altitude, as shown in Fig. 3(c), the performance of most MOT methods decreases with increasing altitude. Our method performs well in low-alt and medium-alt which the appearance difference between objects are various while the scene changes. For Duration, the performance of the proposed method is stable in long-term and short-term that shows the robustness of our method.

\section{CONCLUSIONS}

For online multi-object tracking, the influence of changing context for the discrimination of descriptions are often ignored. We proposed a novel self-balance method fusing appearance similarity and motion consistency to deal with the changing discrimination. Finally, we have tested our method on a large number of datasets and the experimental results show the effectiveness of the proposed online multi-object tracking method.

\section{ACKNOWLEDGMENTS}

This work was supported in part by National Natural Science Foundation of China: 61620106009, 61772494 and 61836002, in part by Youth Innovation Promotion Association CAS, in part by Shandong Provincial Natural Science foundation: ZR2017MF001, in part by the University of Chinese Academy of Sciences.

\section{REFERENCES}

[1] Alexandre Alahi, Kratarth Goel, Vignesh Ramanathan, Alexandre Robicquet, Li Fei-Fei, and Silvio Savarese. 2016. Social lstm: Human trajectory prediction in crowded spaces. In CVPR. 961971.

[2] Keni Bernardin and Rainer Stiefelhagen. 2008. Evaluating Multiple Object Tracking Performance: The CLEAR MOT Metrics. EURASIP J. Image and Video Processing 2008 (2008).

[3] Alex Bewley, ZongYuan Ge, Lionel Ott, Fabio Tozeto Ramos, and Ben Upcroft. 2016. Simple online and realtime tracking. In ICIP. 3464-3468.

[4] Erik Bochinski, Volker Eiselein, and Thomas Sikora. 2017. HighSpeed tracking-by-detection without using image information. In AVSS. $1-6$.

[5] Wongun Choi. 2015. Near-online multi-target tracking with aggregated local flow descriptor. In $I C C V$.

[6] Jifeng Dai, Yi Li, Kaiming He, and Jian Sun. 2016. R-FCN: Object Detection via Region-based Fully Convolutional Networks. In NIPS. 379-387.

[7] Caglayan Dicle, Octavia I. Camps, and Mario Sznaier. 2013. The Way They Move: Tracking Multiple Targets with Similar Appearance. In ICCV. 2304-2311.

[8] Dawei Du, Yuankai Qi, Hongyang Yu, Yifan Yang, Kaiwen Duan, Guorong Li, Weigang Zhang, Qingming Huang, and Qi Tian. 2018. The unmanned aerial vehicle benchmark: Object detection and tracking. In ECCV. 370-386.

[9] Pedro F Felzenszwalb, Ross B Girshick, David McAllester, and Deva Ramanan. 2010. Object detection with discriminatively trained part-based models. IEEE TPAMI 32, 9 (2010), 16271645 .

[10] Ju Hong Yoon, Chang-Ryeol Lee, Ming-Hsuan Yang, and Kuk-Jin Yoon. 2016. Online multi-object tracking via structural constraint event aggregation. In $C V P R$.
[11] Hamid Izadinia, Imran Saleemi, Wenhui Li, and Mubarak Shah. 2012. 2T: Multiple people multiple parts tracker. In ECCV.

[12] Chanho Kim, Fuxin Li, Arridhana Ciptadi, and James M Rehg. 2015. Multiple hypothesis tracking revisited. In $I C C V$.

[13] Chanho Kim, Fuxin Li, and James M Rehg. 2018. Multi-object tracking with neural gating using bilinear lstm. In ECCV. 200215.

[14] Tao Kong, Fuchun Sun, Anbang Yao, Huaping Liu, Ming Lu, and Yurong Chen. 2017. RON: Reverse Connection with Objectness Prior Networks for Object Detection. In $C V P R$.

[15] Wei Liu, Dragomir Anguelov, Dumitru Erhan, Christian Szegedy, Scott E. Reed, Cheng-Yang Fu, and Alexander C. Berg. 2016. SSD: Single Shot MultiBox Detector. In ECCV. 21-37.

[16] Anton Milan, Laura Leal-Taixé, Konrad Schindler, and Ian Reid. 2015. Joint tracking and segmentation of multiple targets. In CVPR.

[17] Anton Milan, Seyed Hamid Rezatofighi, Anthony R Dick, Ian D Reid, and Konrad Schindler. 2017. Online Multi-Target Tracking Using Recurrent Neural Networks.. In $A A A I$.

[18] Anton Milan, Seyed Hamid Rezatofighi, Anthony R. Dick, Ian D. Reid, and Konrad Schindler. 2017. Online Multi-Target Tracking Using Recurrent Neural Networks. In AAAI. 4225-4232.

[19] Anton Milan, Stefan Roth, and Konrad Schindler. 2014. Continuous energy minimization for multitarget tracking. IEEE TPAMI 36,1 (2014), 58-72.

[20] Anton Milan, Stefan Roth, and Konrad Schindler. 2014. Continuous Energy Minimization for Multitarget Tracking. TPAMI 36, 1 (2014), 58-72.

[21] James Munkres. 1957. Algorithms for the assignment and transportation problems. Journal of the society for industrial and applied mathematics 5, 1 (1957), 32-38.

[22] Hamed Pirsiavash, Deva Ramanan, and Charless C. Fowlkes. 2011. Globally-optimal greedy algorithms for tracking a variable number of objects. In CVPR. 1201-1208.

[23] Joseph Redmon, Santosh Divvala, Ross Girshick, and Ali Farhadi. 2016. You only look once: Unified, real-time object detection. In CVPR.

[24] Liangliang Ren, Jiwen Lu, Zifeng Wang, Qi Tian, and Jie Zhou. 2018. Collaborative deep reinforcement learning for multi-object tracking. In ECCV. 586-602.

[25] Shaoqing Ren, Kaiming He, Ross Girshick, and Jian Sun. 2015. Faster R-CNN: Towards real-time object detection with region proposal networks. In Advances in neural information processing systems.

[26] Shaoqing Ren, Kaiming He, Ross B. Girshick, and Jian Sun. 2015. Faster R-CNN: Towards Real-Time Object Detection with Region Proposal Networks. In NIPS. 91-99.

[27] Ergys Ristani, Francesco Solera, Roger S. Zou, Rita Cucchiara, and Carlo Tomasi. 2016. Performance Measures and a Data Set for Multi-target, Multi-camera Tracking. In $E C C V W$. 17-35.

[28] Guang Shu, Afshin Dehghan, Omar Oreifej, Emily Hand, and Mubarak Shah. 2012. Part-based multiple-person tracking with partial occlusion handling. In $C V P R$.

[29] Jeany Son, Mooyeol Baek, Minsu Cho, and Bohyung Han. 2017. Multi-Object Tracking with Quadruplet Convolutional Neural Networks. In CVPR.

[30] Siyu Tang, Bjoern Andres, Mykhaylo Andriluka, and Bernt Schiele. 2016. Multi-Person Tracking by Multicut and Deep Matching. In $E C C V$.

[31] Siyu Tang, Mykhaylo Andriluka, Bjoern Andres, and Bernt Schiele. 2017. Multiple People Tracking by Lifted Multicut and Person Re-identification. In $C V P R$

[32] Shangzhi Teng, Xiaobin Liu, Shiliang Zhang, and Qingming Huang. 2018. SCAN: Spatial and Channel Attention Network for Vehicle Re-Identification. In Pacific Rim Conference on Multimedia. Springer, 350-361.

[33] Fan Yang, Wongun Choi, and Yuanqing Lin. 2016. Exploit all the layers: Fast and accurate cnn object detector with scale dependent pooling and cascaded rejection classifiers. In $C V P R$.

[34] Ju Hong Yoon, Ming-Hsuan Yang, Jongwoo Lim, and Kuk-Jin Yoon. 2015. Bayesian Multi-object Tracking Using Motion Context from Multiple Objects. In WACV. 33-40.

[35] Hongyang Yu, Lei Qin, Qingming Huang, and Hongxun Yao. 2018. Online multiple object tracking via exchanging object context. Neurocomputing 292 (2018), 28-37.

[36] Ji Zhu, Hua Yang, Nian Liu, Minyoung Kim, Wenjun Zhang, and Ming-Hsuan Yang. 2018. Online multi-object tracking with dual matching attention networks. In ECCV. 366-382. 